\title{
Improved molecular fingerprint analysis employing multi-branched gold nanoparticles in conjunction with surface-enhanced Raman scattering
}

This article was published in the following Dove Press journal:

International Journal of Nanomedicine

22 December 2015

Number of times this article has been viewed

\author{
Jencilin Johnston' \\ Erik N Taylor ${ }^{1,2}$ \\ Richard J Gilbert ${ }^{2}$ \\ Thomas J Webster ${ }^{1,3}$ \\ 'Department of Chemical Engineering, \\ ${ }^{2}$ Department of Chemistry and \\ Chemical Biology, Northeastern \\ University, Boston, MA, USA; ${ }^{3}$ Center \\ of Excellence for Advanced Materials \\ Research, King Abdulaziz University, \\ Jeddah, Saudi Arabia
}

\begin{abstract}
Vibrational spectroscopy is a powerful analytical tool that assesses molecular properties based on spectroscopic signatures. In this study, the effect of gold nanoparticle morphology (spherical vs multi-branched) was assessed for the characterization of a Raman signal (ie, molecular fingerprint) that may be helpful for numerous medical applications. Multibranched gold nanoparticles (MBAuNPs) were fabricated using a green chemistry method which employed the reduction of gold ion solute by 2-[4-(2-hydroxyethyl)-1-piperazyl] ethane sulfonic acid. Two types of reporter dyes, indocyanine (IR820 and IR792) and carbocyanine (DTTC [3, '3'-diethylthiatricarbocyanine iodide] and DTDC [3,3'-diethylthiadicarbocyanine iodide]), were functionalized to the surface of the MBAuNPs and stabilized with denatured bovine serum albumin, thus forming the surface-enhanced Raman spectroscopy tag. Fluorescein isothiocyanate-conjugated anti-epidermal growth factor receptor to the surface-enhanced Raman spectroscopy tags and the properties of the resulting conjugates were assessed through determination of the Raman signal. Using the MBAuNP Raman probes synthesized in this manner, we demonstrated that MBAuNP provided significantly more surface-enhanced Raman scattering signal when compared with the associated spherical gold nanoparticle of similar size and concentration. MBAuNP enhancements were retained in the surface-enhanced Raman spectroscopy tags complexed to anti-epidermal growth factor receptor, providing evidence that this could be a useful biological probe for enhanced Raman molecular fingerprinting. Furthermore, while utilizing IR820 as a novel reporter dye linked with MBAuNP, superior Raman signal fingerprint results were obtained. Such results provide significant promise for the use of MBAuNP in the detection of numerous diseases for which biologically specific surface markers exist.
\end{abstract}

Keywords: gold nanostars, IR820, SERS

\section{Introduction}

The discovery by Sir CV Raman that light energy is scattered by the substance onto which it is incident was termed the Raman Effect and was the basis for the Nobel Prize in 1930 in physics. When the light energy, E=hv, is incident on a molecule, it is scattered with the same amount of energy, called Rayleigh scattering. If the energy of the scattered light is lost or gained, generally due to molecular vibrations, it is called inelastic scattering or Raman scattering. If the scattered light has lost energy from that of the incident light, it is called Stokes Raman scattering and if it has gained energy it is called anti-Stokes Raman scattering (Figure 1). Since this vibrational effect is unique for each molecule, the Raman effect may be considered as a molecular fingerprint. However, the Raman signal is very weak, where only one photon out of $10^{10}$ incident photons is scattered. ${ }^{1}$ Surface-enhanced Raman scattering (SERS) has been employed as a method to enhance Raman signals in the order of $10^{12}$ to $10^{14}$.
Correspondence: Thomas J Webster Department of Chemical Engineering, Northeastern University, 313 Snell Engineering, 360 Huntington Avenue, Boston, MA 02115, USA

Email th.webster@neu.edu 
A
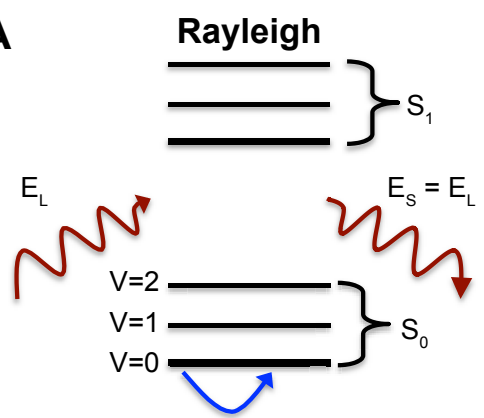

C

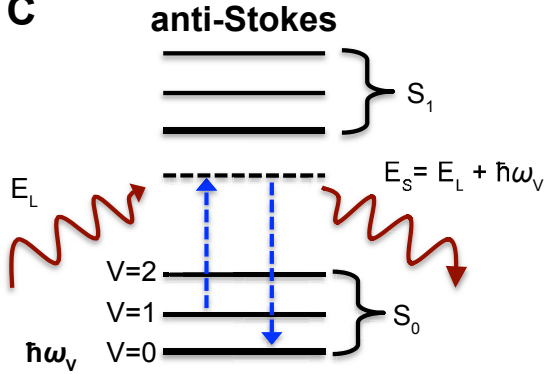

B

Raman
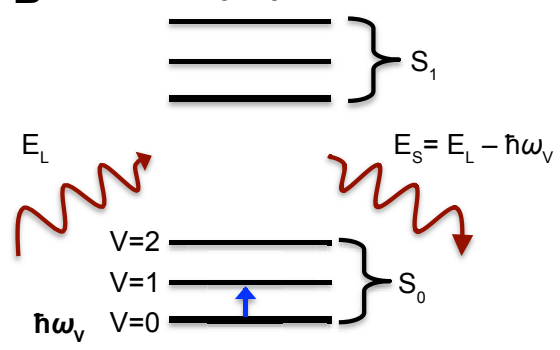

D

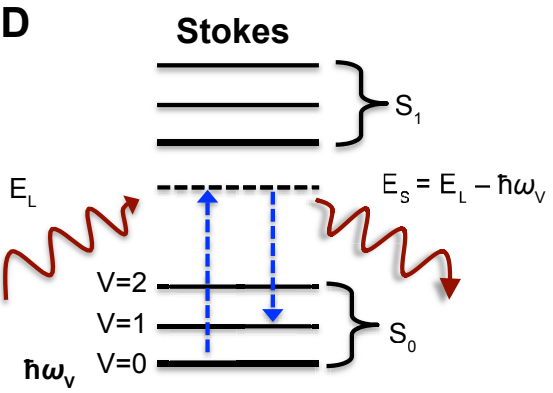

Figure I Jablonski energy diagram for Rayleigh scattering (A), linear spontaneous Raman scattering (B), anti-Stokes Raman scattering (C), and Stokes Raman scattering (D), Abbreviations: $\mathrm{S}_{0}$, ground state; $\mathrm{S}_{\mathrm{l}}$, excited state; $\mathrm{E}_{\mathrm{L}}$, energy of incident light; $\mathrm{E}_{\mathrm{s}}$, scattered energy; $\hbar \omega_{\mathrm{v}}$, vibrational energy.

SERS is a nondestructive, ultrasensitive, reproducible, inelastic vibrational scattering technique which gives accurate fingerprints of the molecules of interest. ${ }^{2}$ Surface plasmonic resonance results from the oscillation of free electrons in conductive metals like copper, silver, and gold, when excited by the incident light energy. Localized surface plasmon resonance occurs when these oscillations are confined to the nanostructure. ${ }^{3}$ Localized surface plasmon resonance underlies the SERS effect with the promotion of oscillatory effects in free electrons on the surface of the nanoparticles and through the generation of higher dipole moments in these electrons, thus creating "hotspots" ${ }^{4}$ When reporter molecules are adherent on the hotspots, the localized surface plasmon resonance effect caused by the electrons induces a dipole moment on the molecules adsorbed on the nanoparticles which causes the molecules to polarize, thereby setting the adherent molecules into vibration. Since vibrational scattering is unique for each molecule, this method gives an accurate result of the analyte, thereby giving the molecular fingerprint of the analyte. Gold, which has good biocompatible properties and good optical properties, ${ }^{5}$ has been utilized to enhance the weak Raman scattering signal emitted by the analyte when irradiated with the monochromatic laser light.

In this study, we synthesized multi-branched gold nanoparticles (MBAuNPs) and adhered two different kinds of Raman reporters, indocyanine and carbocyanine dyes, to identify the dye that yields maximum Raman intensity. A comparative study on the enhancement effect of MBAuNP and spherical AuNP was performed and a relative enhancement factor of MBAuNP was determined and calculated. Such results provided significant promising data for the use of MBAuNP (as compared to AuNPs) in the detection and treatment of numerous diseases from cancer to cardiovascular disease.

\section{Materials and methods Materials}

Hydrotetracholoaurate $\left(\mathrm{HAuCl}_{4}\right), 2$-[4-(2-hydroxyethyl)1-piperazyl] ethane sulfonic acid (HEPES), IR820, IR792, DTTC (3,3'-diethylthiatricarbocyanine iodide), DTDC (3,3'-diethylthiadicarbocyanine iodide), and sodium hydroxide $(\mathrm{NaOH})$ were bought from Sigma-Aldrich Co., St Louis, MO, USA. Spherical AuNPs $60 \mathrm{~nm}$ in diameter were bought from Cytodiagnostics, ON, Canada. The monoclonal anti-epidermal growth factor receptor (EGFR) antibody conjugated with fluorescein isothiocyanate (FITC) was bought from Abcam, Cambridge, MA, USA. Sodium bicarbonate was purchased from Thermo Fisher Scientific, Waltham, MA, USA.

\section{Synthesis of MBAuNP}

MBAuNP were prepared at room temperature at $\mathrm{pH} 7.4$ using a seedless, green chemistry method ${ }^{6}$ in which $\mathrm{HAuCl}_{4}$ was reduced by the HEPES. All the glassware were washed with 
aqua regia, ethanol, and deionized water. A 15 mM HEPES solution was prepared by adding $1.5 \mathrm{~mL}$ of $100 \mathrm{mM}$ HEPES to $8.5 \mathrm{~mL}$ of deionized water. The $\mathrm{pH}$ of the solution was adjusted to 7.4 by the addition of $1 \mathrm{M} \mathrm{NaOH}$. Then, $0.25 \mathrm{~mL}$ of $20 \mathrm{mM} \mathrm{HAuCl}_{4}$ was added and the colorless solution turned turbid blue within 30 to 45 minutes.

\section{Characterization of MBAuNP}

The synthesized MBAuNPs were characterized using a SpectraMax Paradigm to obtain the absorption spectrum of these particles and a JOEL transmission electron microscope (TEM) 1010 was used to obtain the size and shape of the nanoparticles. For this, $10 \mu \mathrm{L}$ of the washed MBAuNP was placed on a neat parafilm and the copper grid was placed on the droplet and dried to remove excess solution. Then, they were scanned using a JEOL TEM 1010. A Hitachi 4800 Scanning Electron Microscope was used to obtain the morphology of the nanoparticles. For this, a sample holder was coated with a palladium coating and the MBAuNPs were placed on the coated sample holder and dried; then, it was scanned in the scanning electron microscope. Lastly, a DLS 90 Plus Brookhaven instrument was used to obtain the hydrodynamic size of the particles.

\section{Preparation of SERS tags}

A stock solution of a $1 \mathrm{mM}$ concentration of the Raman probes, DTTC, DTDC, IR792, and IR820, was prepared. An amount of $5 \mu \mathrm{M}$ of each of the Raman probe solutions was prepared by the addition of $5 \mu \mathrm{L}$ of the Raman probe solution to $995 \mu \mathrm{L}$ of deionized water. Raman probe solutions of varying volumes from 1 to $10 \mathrm{~mL}$ were added to 1 $\mathrm{mL}$ of colloidal MBAuNP solution by vortexing at medium speed and incubating for 10 minutes. Then, the excess Raman probes were removed by centrifuging at 3,000 rpm for 10 minutes. The centrifuged MBAuNP were then incubated in $0.5 \mathrm{mg} / \mathrm{mL}$ of denatured bovine serum albumin (dBSA) for 1 hour. The excess dBSA was removed by centrifuging three times at 3,000 rpm for 10 minutes and by rinsing with deionized water.

\section{Raman spectra of Raman probes}

The Raman spectra of the different Raman probes, DTTC, DTDC, IR792, and IR820, were obtained using a Horiba Jobin Yvon LabHR800 instrument with power of $20 \mathrm{~mW}$ at $10 \times$ magnification. The instrument was first calibrated for the silicon peak at $520 \mathrm{~cm}^{-1}$. An amount of $20 \mu \mathrm{L}$ of the samples was placed on the fused silica slide and irradiated with a red laser at a wavelength of $785 \mathrm{~nm}$.

\section{Conjugation of SERS tag with antibodies}

The SERS tag of the Raman probe, IR820, was conjugated with the FITC-conjugated antibody through an ethyl dimethylaminopropyl carbodiimide-N-hydroxysuccinimide coupling reaction. ${ }^{7}$ An amount of $5 \mu \mathrm{L}$ of $0.2 \mathrm{M}$ freshly prepared ethyl dimethylaminopropyl carbodiimide and $10 \mu \mathrm{L}$ of $0.2 \mathrm{M}$ freshly prepared $\mathrm{N}$-hydroxysuccinimide was added to the SERS tags suspended in $15 \mathrm{mM}$ HEPES and gently mixed for 3 minutes. The excess ethyl dimethylaminopropyl carbodiimide-N-hydroxysuccinimide was removed by centrifuging three times with $15 \mathrm{mM}$ HEPES at 3,000 rpm for 10 minutes. Then, the FITC-conjugated anti-EGFR was reacted with the carboxyl-activated SERS tags for 2 hours at room temperature and then at $4^{\circ} \mathrm{C}$ overnight. The excess and unbound antibodies were removed by three rounds of centrifugation at 3,000 rpm for 10 minutes.

All the experiments were repeated three times.

\section{Results and discussion Synthesis of MBAuNP}

HEPES is a zwitter ion. At pH 7.4, the HEPES buffer, which has piperazine groups, produces nitrogen-centered free radicals that reduce the trivalent gold ions to divalent gold ions and monovalent gold ions, and finally into gold zerovalent ions which is due to the aggregation of the reduced gold ions. This reaction proceeds until the availability of gold ions and after that the HEPES acts as a stabilizing agent. The salt $\mathrm{HAuCl}_{4}$ contains $\mathrm{Au}^{3+}$ ions, and these ions are reduced by the free radicals generated by HEPES at $\mathrm{pH}$ 7.4. Since the availability of these $\mathrm{Au}^{3+}$ ions is limited, the reduction continues until their availability. ${ }^{8}$ Per the literature, the mechanism for the redox reaction is:

$$
\begin{aligned}
& \mathrm{Au}(\mathrm{III})+\mathrm{HEPES} \rightarrow \mathrm{Au}(\mathrm{II})+\text { cationic free radicals } \\
& 2 \mathrm{Au}(\mathrm{II}) \rightarrow \mathrm{Au}(\mathrm{III})+\mathrm{Au}(\mathrm{I}) \\
& \mathrm{Au}(\mathrm{I})+\mathrm{HEPES} \rightarrow \mathrm{Au}(0)+\text { cationic radicals } \\
& \mathrm{n} \mathrm{Au}(0) \rightarrow(\mathrm{Au}(0))_{\mathrm{n}}\{\mathrm{AuNPs}\} \\
& \text { Cationic free radicals }+\mathrm{O}_{2} \rightarrow \text { degraded products. }
\end{aligned}
$$

\section{Characterization of MBAuNP}

The ultraviolet-visible spectra of the MBAuNP showed the presence of two peaks: a transverse plasmonic ${ }^{8}$ resonance of a small peak at $530 \mathrm{~nm}$, confirming the presence of a spherical core, and a longitudinal plasmonic resonance ${ }^{8}$ of broad peak at $665 \mathrm{~nm}$, confirming the presence of the branched shaped aggregation on the spherical core (Figure 2). The broad red shift from 630 to $700 \mathrm{~nm}$ is caused by the collective oscillation of free electrons (the surface plasmonic effect) on the 


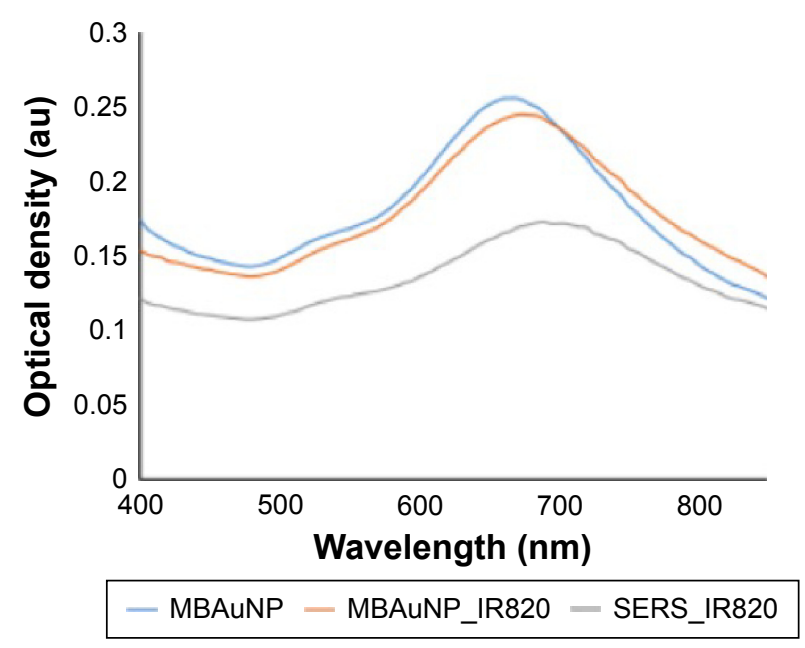

Figure 2 UV-vis spectrum of an MBAuNP showing two peaks at 530 and $665 \mathrm{~nm}$ indicating a spherical core and branches, respectively.

Abbreviations: UV-vis, ultraviolet-visible; MBAuNP, multi-branched gold nanoparticle.

aggregated gold ions, which were present on the branches of the MBAuNP.

The ultraviolet-visible spectrum of MBAuNP was confirmed by the TEM images, which confirmed the presence of the spherical core surrounded with 12 to 14 numbers of branches with varying lengths from 8 to $29 \mathrm{~nm}$. The size of MBAuNP ranged from $55 \mathrm{~nm}$ to $65 \mathrm{~nm}$ (Figure 3 ). The more the number of branches, the higher the oscillation of free electrons on the surface, causing a plasmonic effect, which is the underlying cause for the SERS effect. This was further confirmed by the scanning electron microscope image, which showed a uniform morphology of MBAuNP and provides an insight into 3D orientation of distributed branched structures (Figure 4). The bright fields on the edges of the MBAuNP were due to the aggregated Au ions on the core.

The DLS data showed that the MBAuNP, MBAuNP_ IR820, and SERS_IR820 tag was 69.8, 76.7, and $79.2 \mathrm{~nm}$, respectively. The increase in the hydrodynamic diameter indicates the presence of reporter IR820 on the MBAuNP and the encapsulation of dBSA of the same as shown in Figure 5.

\section{Characterization of SERS tags}

The Raman spectra of the Raman probes functionalized on the MBAuNP showed the maximum Raman intensity of 213.6, 598.5, 1,020.52, and 1,999.21 for DTDC, DTTC, IR792, and IR820, respectively (Figure 6). Since IR820 exhibited maximum Raman intensity, we prepared SERS tags with an IR820 probe for further experiments.

Bovine serum albumin, which is generally used as a stabilizing agent, has 35 thiol (-SH) groups of which 34 are involved in the formation of disulfide bonds leaving one free thiol group. These $-\mathrm{SH}$ are liberated by denaturing the bovine serum albumin, thereby making more - $\mathrm{SH}$ groups available for conjugating with MBAuNP. Moreover, dBSA is used as a stabilizing agent due to its ability to quench the fluorescence. ${ }^{9}$ The dBSA attached to the MBAuNP through cysteine bonds and were formed by reducing bovine serum albumin with sodium borohydride. ${ }^{6}$ These free thiol (-SH) groups, which possess a high affinity toward AuNP surfaces, were used to bind dBSA to the nanoparticle while making available carboxyl groups for the attachment of the antibody.

Raman spectra of the SERS tag with IR820, MBAuNP with IR820, and IR820 solution without MBAuNP are shown
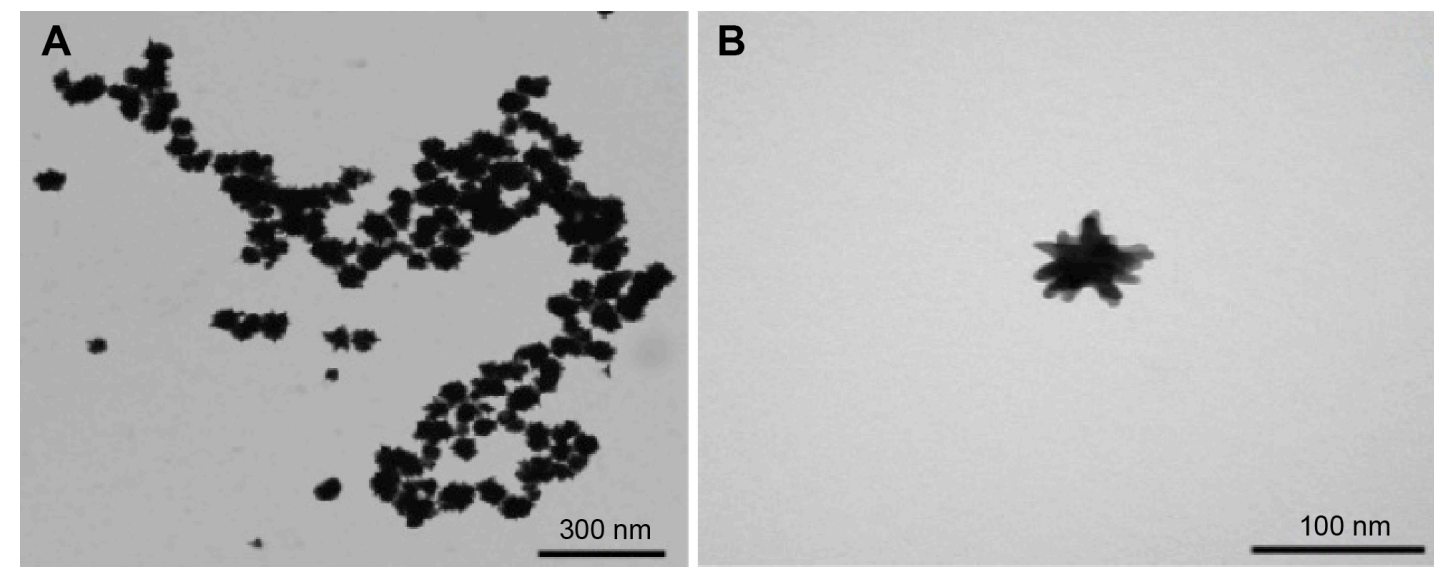

Figure 3 TEM images of an MBAuNP.

Note: (A) 2D distribution of MBAuNPs with their branches and (B) 2D view of a single MBAuNP with I2 to I4 branches.

Abbreviations: TEM, transmission electron microscope; MBAuNP, multi-branched gold nanoparticle; 2D, two dimensional. 


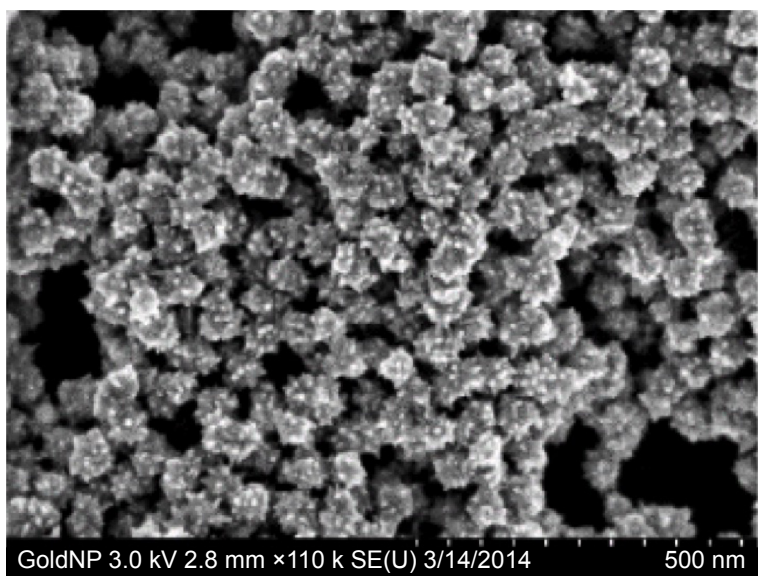

Figure 4 SEM image of an MBAuNP: 3D view of the uniformly distributed MBAuNP.

Notes: The glow on the branches shows that there is much electron density, causing the LSPR effect as they were irradiated by the electron beam.

Abbreviations: SEM, scanning electron microscope; MBAuNP, multi-branched gold nanoparticle; LSPR, localized surface plasmon resonance; 3D, three dimensional.

in Figure 7. The encapsulation of functionalized MBAuNP with IR 820 by dBSA showed a decrease in the Raman intensity, as shown in Figure 7.

\section{Fingerprint of IR820}

The IR820 has two indole rings, separated by a polymethine chain with one benzyl chloride at the middle of the chain, and the entire chain is terminated by benzene rings at the ends ${ }^{10,11}$ as shown in the inset of Figure 8.

The peaks were analyzed with reference to the study completed by Gediminas Niaura et al and Giuseppe Perna et al on Fourier Transform-Raman spectrum for N-[2-(1H-indol-3-yl)ethyl]-8-mercaptooctanamide ${ }^{12}$ and

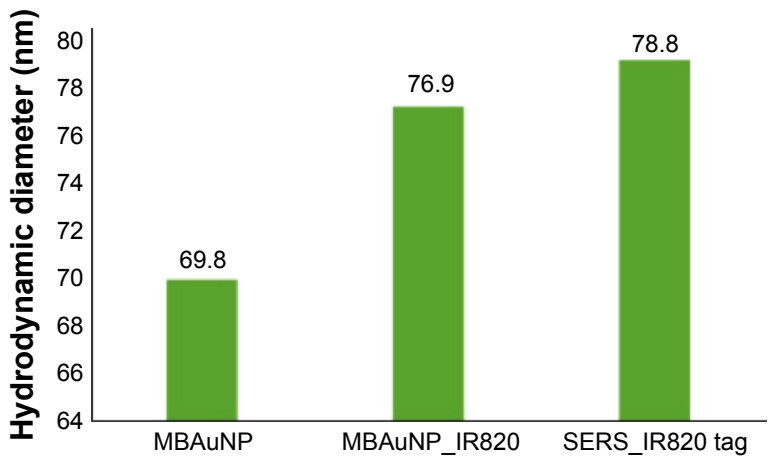

Figure $\mathbf{5}$ The hydrodynamic diameter using DLS showing that with a successive synthesis step in the preparation of MBAuNP, MBAuNP_IR820, and SERS_IR820, increased diameters were observed.

Notes: The change in diameter from MBAuNP to MBAuNP_IR820 is through the addition of IR820 molecules resulting in the diameter change of $7.1 \mathrm{~nm}$. The change in diameter $(1.9 \mathrm{~nm})$ from the MBAuNP_IR820 to SERS_IR820 tag is through the addition of dBSA.

Abbreviations: MBAuNP, multi-branched gold nanoparticle; SERS, surface-enhanced Raman spectroscopy; dBSA, denatured bovine serum albumin; DLS, dynamic light scattering.

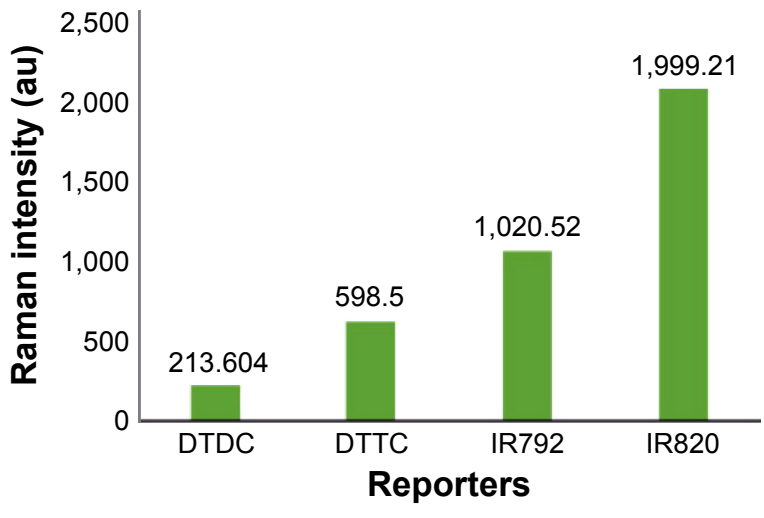

Figure 6 Raman intensity of Raman probes, DTDC, DTTC, IR792, and IR820.

vibrational characterization for Eumelanin. ${ }^{13}$ The SERS peak at $1,523.07 \mathrm{~cm}^{-1}$ corresponds to the pyrrole stretching, $1,626.78 \mathrm{~cm}^{-1}$ corresponds to the superposition of in-plane benzene and pyrrole stretching mode, $1,446.21 \mathrm{~cm}^{-1}$ corresponds to $-\mathrm{NH}$ deformation and benzene deformation. The $\mathrm{C}-\mathrm{N}$ bond stretching of the pyrrole ring at $1,359.74 \mathrm{~cm}^{-1}$ and Fermi resonance effect was observed at 1,127.79, 1,147.69, and $1,168.97 \mathrm{~cm}^{-1}$ for the $-\mathrm{CH}$ bond of the benzene ring and $\mathrm{C}-\mathrm{C}$ bonds from the backbone structure of polymethine at $1,228.28$ and $1,264.35 \mathrm{~cm}^{-1}$. Thus, the fingerprint of the IR 820 was obtained by the SERS effect clearly showing the backbone, polymethine structure, indole, and benzene rings.

\section{Comparison of MBAuNP SERS tags with spherical AuNP SERS tags}

The Raman intensity enhancement of the MBAuNP SERS tags was studied and compared with those of the spherical AuNP. A SERS tag of spherical AuNP was prepared by the method similar to that explained in the "Materials and methods" section. Though the spherical particles enhanced

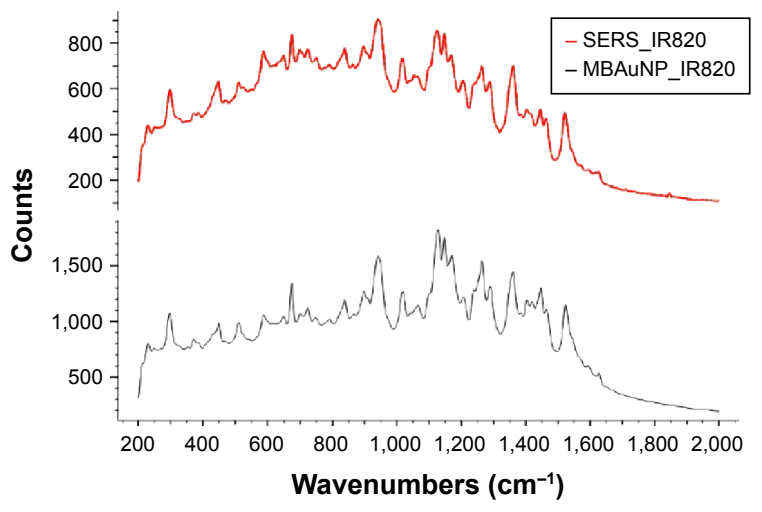

Figure 7 Raman spectra of an MBAuNP with the IR820 and SERS tag IR820 showing the decrease in intensity.

Abbreviations: MBAuNP, multi-branched gold nanoparticle; SERS, surface-enhanced Raman spectroscopy. 


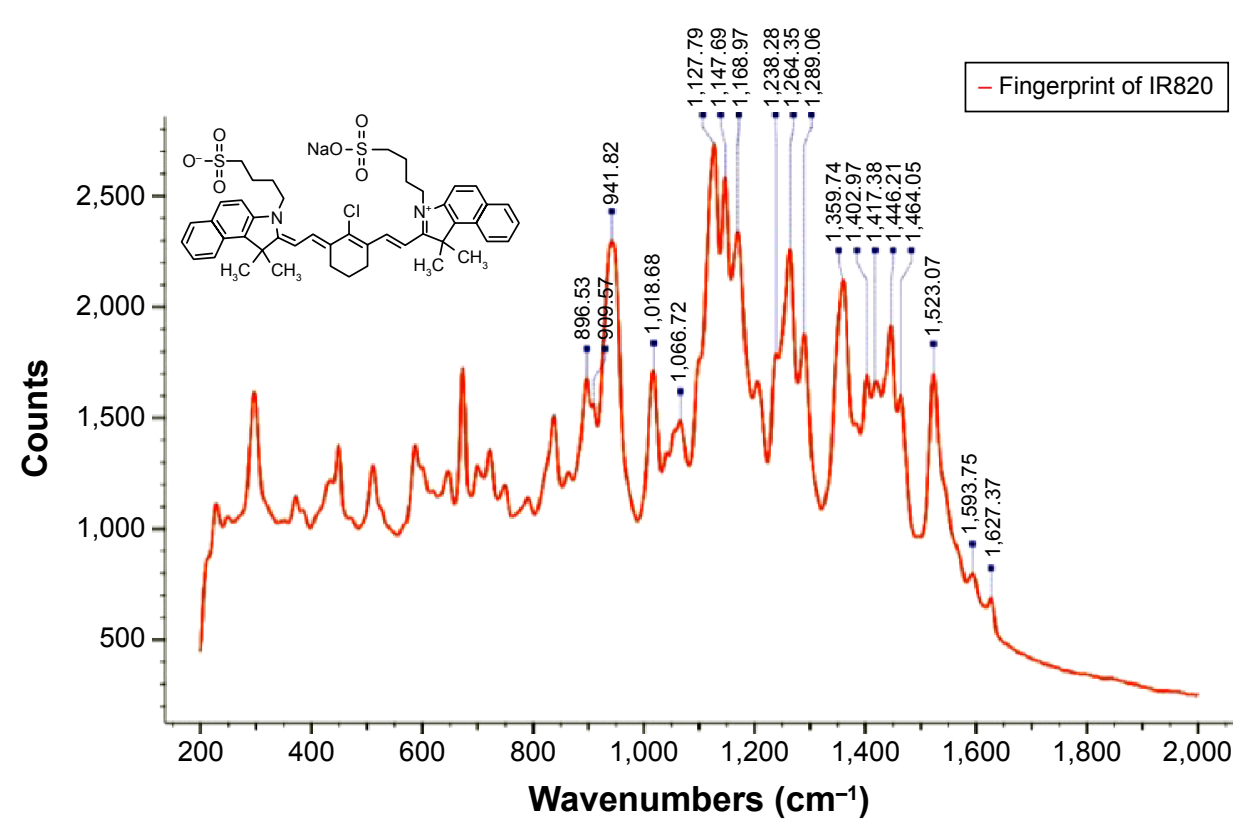

Figure 8 Fingerprint of IR820; the inset shows the chemical structure of IR820.

Note: The SERS effect clearly shows the bond deformation of the molecules in IR820.

Abbreviation: SERS, surface enhanced Raman spectroscopy.

the Raman signal, it was much less when compared to that of the MBAuNP. Moreover, the number of spherical AuNPs per $\mathrm{mL}, 1.957 \times 10^{10}$, was greater than that of the MBAuNP per $\mathrm{mL}, 1.577 \times 10^{10}$; the number of particles was calculated in reference with Khlebtsov ${ }^{14}$ and is shown in the Supplementary materials (Section 1). So, the results of this study showed that it is the shape of the nanoparticles that plays a vital role in the SERS effect more than the number of particles. The fingerprint region of the IR820 was clearly visible for the MBAuNP than that of the spherical particles (Figure 9). The Raman scattering signal enhancement factor of the MBAuNP was calculated as $4.028 \times 10^{4}$; the calculation for SERS enhancement factor is shown in Supplementary materials (Section 2).

\section{FITC-conjugated anti-EGFR with SERS tag}

The conjugation of FITC-conjugated anti-EGFR was confirmed with the aid of a Carl-Zeiss confocal microscope. The antibody attached particles aggregated to enable easy visualization through the confocal microscope. The SERS tags with antibody showed green fluorescence from FITC (Figure 10).

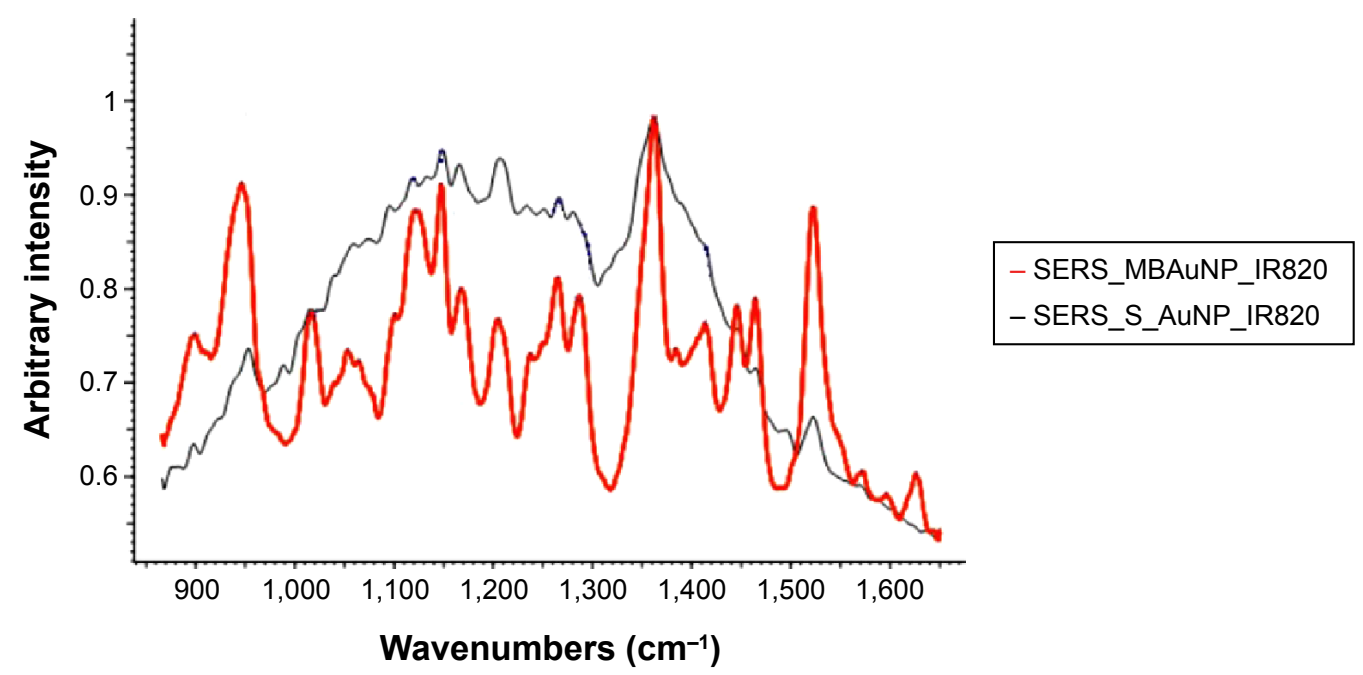

Figure 9 A comparative study of Raman spectra of SERS_MBAuNP_IR820 tag and SERS_Spherical_IR820 tag: the spherical nanoparticle enhances the Raman signal from IR820 but is much less than that of MBAuNP.

Abbreviations: MBAuNP, multi-branched gold nanoparticle; SERS, surface-enhanced Raman scattering. 

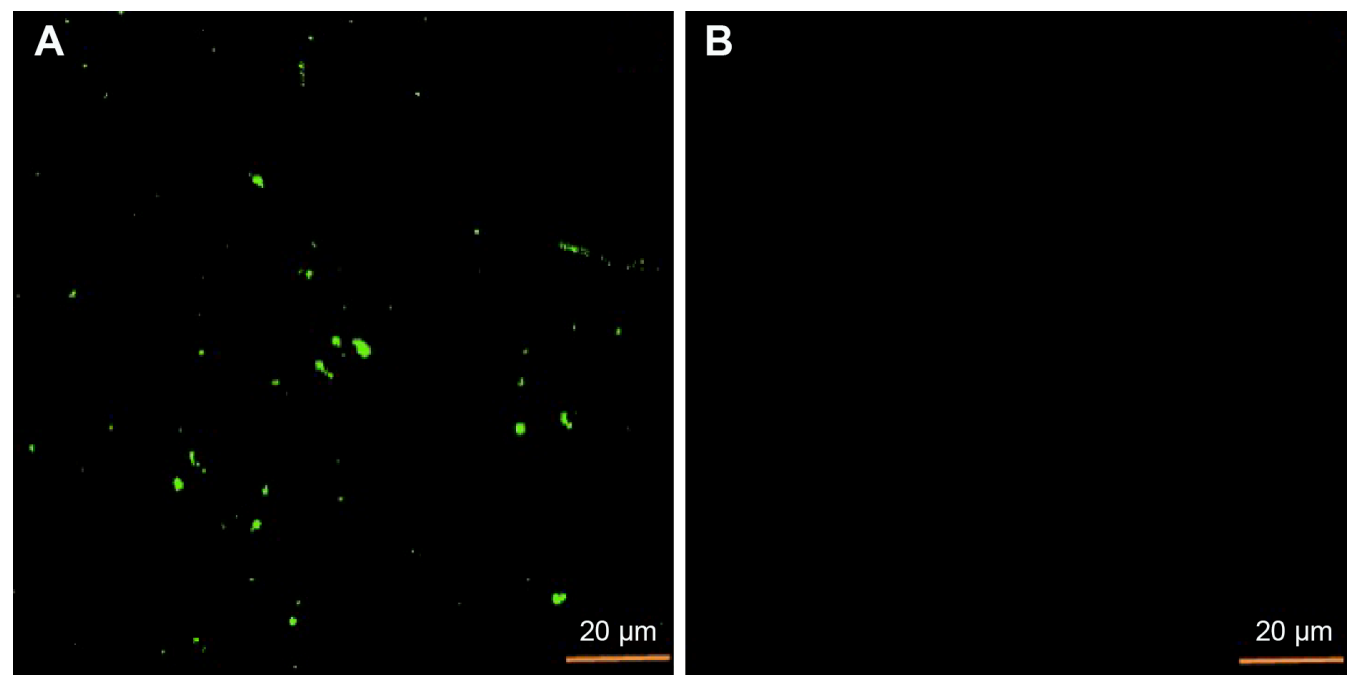

Figure 10 Fluorescence image of SERS tags.

Notes: (A) Shows the green fluorescence of the FITC-conjugated Ab conjugated to the SERS tags and (B) shows that of the control without an antibody attached on the SERS tags.

Abbreviations: SERS, surface-enhanced Raman spectroscopy; FITC, fluorescein isothiocyanate.

\section{Conclusion}

Results demonstrate that MBAuNP enhanced the Raman signal of IR820, with and without complexation to antiEGFR antibody, considerably more than the enhancement seen with spherical AuNPs. The enhancement factor of MBAuNP was $4.028 \times 10^{4}$. This study demonstrates promise for the use of MBAuNP complexed with various antibodies in the detection and characterization of various diseases with discernible surface markers. Future studies will extend these findings by employing MBAuNP-enhanced SERS to detect EGFR, and similar marker substances, on the surface of cancer cells.

\section{Acknowledgment}

The authors would like to thank Northeastern University for sponsoring this study.

\section{Disclosure}

The authors report no conflicts of interest in this work.

\section{References}

1. Hao F, Nehl C, Hafner J, Norlander P. Plasmon resonances of gold nanostar. Nano Lett. 2007;7(3):729-732.

2. Wang Y, Yan B, Chen L. SERS tags: novel optical nanoprobes for bioanalysis. ACS Chem Rev. 2013;113(3):1391-1428.
3. Elsevier. Raman spectroscopy and related techniques. Available form www.knovel.com. Accessed October 19, 2015.

4. Alvarez-Puebla RA, Liz-Marzán LM. SERS-based diagnosis and biodetection. Small. 2010;6(5):604-610.

5. Yigit MV, Medarova Z. In vivo and ex vivo applications of gold nanoparticles for biomedical SERS imaging. $\mathrm{Am} \mathrm{J} \mathrm{Nucl} \mathrm{Med} \mathrm{Mol}$ Imaging. 2012;2(2):232-241.

6. Xie J, Zhang Q, Lee J, Wang D. The synthesis of SERS-active gold nanoflower tags for in vivo applications. ACS Nano. 2008;2(12) 2473-2480.

7. Bartczak D, Kanaras A. Preparation of peptide-functionalized gold nanoparticles using one pot EDC/sulfo-Nhs coupling. Langmuir. 2011; 27(16):10119-10123.

8. Habib A, Tabata M, Wu Y. Formation of gold nanoparticles by Good's buffer. Chem Soc Jpn. 2005;78:262-269.

9. Li H, Guo Y, Xiao L, Chen B. Selective and sensitive detection of acetyl cholinesterase activity using denatured protein protected gold nanoclusters as a label-free probe. Analyst. 2014;139:285-289.

10. Fernandez-Fernandez A, Manchanda R, Lei T, et al. Comparative study of the optical and heat generation properties of IR 820 and indocyanine green. Mol Imaging. 2012;11(2):99-113.

11. Fernandez-Fernandez A, Manchanda R, Lei T, Srinivasan S, McGoron AJ. IR820 conjugates for image-guided cancer therapy. IFMBE Proc. 2013;39:1636-1639.

12. Razmute I, Kuodis Z, Eicher-Lorka O, Niaura G. Surface enhanced Raman spectroscopy of indole ring terminated self assembled monolayer on silver electrode. CHEMIJA. 2006;17(1):25-30.

13. Perna G, Lasalvia M, Gallo C, Quartucci G, Capozzi V. Vibrational characterization of synthetic Eumelain by means of Raman and Surface Enhanced Raman Scattering. Open Surf Sci J. 2013;5:1-8.

14. Khlebtsov N. Determination of size and concentration of gold nanoparticles from extinction spectra. Anal Chem. 2008;80(17): $6620-6625$. 


\section{Supplementary materials Section I}

The diameter of multi-branched gold nanoparticle (MBAuNP) was calculated using the Mie theory for Maxwell's equations, which gives the extinction and scattering efficiency of small nanoparticles. In accordance with Khlebtsov, ${ }^{14}$ the particle diameter was calculated from ultraviolet-visible spectra. The explanation is as follows.

For the particles whose diameter, d, varies from 35 to $100 \mathrm{~nm}$ :

$$
\mathrm{d}=\frac{\ln \frac{\lambda_{s p r}-\lambda_{e}}{L_{1}}}{L_{2}}
$$

for $\mathrm{d}>25 \mathrm{~nm}$, the theoretical fit parameters were $\lambda_{0}=512$, $L_{1}=6.53$, and $L_{2}=0.216$.

For spherical gold nanoparticles:

$$
\mathrm{d}=\frac{\ln \frac{(540-512)}{6.53}}{0.0216}
$$

$\mathrm{d}=67.39 \mathrm{~nm}$.

The number density of the particle is given by the equation:

$$
\mathrm{N}=\frac{A_{450} * 10^{14}}{d^{2}\left[-0.295+1.36 \exp \left(-\left(\frac{d-96.8}{78.2}\right)^{2}\right)\right]}
$$

The Beer's law, $A=\varepsilon^{*} C^{*} 1$,

where

$\mathrm{A}$ is the absorbance

$\mathrm{C}$ is the concentration of the nanoparticle

1 is the path length.

For $\mathrm{l}=1 \mathrm{~cm}$ and area of the micro-well plate to be $0.56 \mathrm{~cm}^{2}$.

For $A_{450}=0.3125, \mathrm{~d}=60 \mathrm{~nm}$, the number of spherical particle was found to be $1.957 \times 10^{10} / \mathrm{mL}$ which is $\sim$ equal to that of the vendors quote.

In a similar way, the number of $\mathrm{MBAuNP} / \mathrm{mL}$ was calculated to be:

$$
\mathrm{N}_{\mathrm{MBAuNP}}=1.577 \times 10^{10} / \mathrm{mL}
$$

\section{Section 2}

The surface-enhanced Raman spectroscopy (SERS) enhancement factor can be determined using the following equation:

$$
\mathrm{EF}=\frac{I_{S E R S}}{I_{N R S}} * \frac{N_{N R S}}{N_{S E R S}}
$$

where

$\mathrm{EF}$ is the enhancement factor

$I_{S E R S}$ and $I_{N R S}$ are the SERS intensity and normal Raman intensity, respectively

$N_{S E R S}$ and $N_{N R S}$ are the number of particles probed for SERS and normal Raman setting.

An amount of $20 \mu \mathrm{L}$ of a $5 \mu \mathrm{M}$ IR 820 solution and $20 \mu \mathrm{L}$ of multi-branched gold nanoparticle (MBAuNP)_IR820 were studied under identical conditions: laser power of $20 \mathrm{~mW}$ and $785 \mathrm{~nm}$ wavelength at $10 \times$ magnification.

The number of particles (IR820) adhered on the MBAuNP was calculated using optical density; it was found that there were 901 molecules adsorbed on one MBAuNP.

There were $1.577 \times 10^{10}$ MBAuNPs in $1 \mathrm{~mL}$ (calculated using ultraviolet spectrum).

Since the enhancements are more at hotspots which are the branches for this MBAuNP, assuming the branches to be the triangle and from the transmission electron microscope image, the width of branch $=7 \mathrm{~nm}$ and the length of the branch $=20 \mathrm{~nm}$.

The area of the hotspot $=1 / 2 *$ base $*$ height $=70 \mathrm{~nm}^{2}$.

The transmission electron microscope image shows that the number of branches varies from 12 to 14 ; assuming there were 12 branches on one MBAuNP:

The total surface area available for the IR 820 to be adsorbed $=12 * 70=840 \mathrm{~nm}^{2}$.

The total surface area of the hotspots/surface area of the spherical core $=840 /\left(\pi * 30^{2}+840\right)=0.229$.

The total number of particles adherent on the hotspots in one MBAuNP $=0.229 * 901=206.3$ molecules of IR820.

There were $6.3 \times 10^{8}$ MBAuNPs in $20 \mu \mathrm{L}$, so the number of IR820 molecules adhered on these MBAuNP, $N_{\text {SERS }}=1.3013 \times 10^{11}$ molecules.

$N_{N R S}$, the number molecules probed for normal Raman $=6.023 * 10^{13}$.

The peak at $1,127.79 \mathrm{~cm}^{-1}$ was considered for this study because it represents the $-\mathrm{CH}$ bond stretching of the benzene ring.

$I_{S E R S}$ at $1,127.79 \mathrm{~cm}^{-1}$ is $1,404.14$ units.

$I_{N R S}$ at $1,127.79 \mathrm{~cm}^{-1}$ is 734.06 units.

$$
\mathrm{EF}=\frac{63,897.78}{734.06} * \frac{6.023 * 10^{13}}{1.3013 * 10^{11}}
$$

$\mathrm{EF}=4.028 * 10^{4}$ 
International Journal of Nanomedicine

Dovepress

\section{Publish your work in this journal}

The International Journal of Nanomedicine is an international, peerreviewed journal focusing on the application of nanotechnology in diagnostics, therapeutics, and drug delivery systems throughout the biomedical field. This journal is indexed on PubMed Central, MedLine, CAS, SciSearch ${ }^{\circledR}$, Current Contents ${ }^{\circledR} /$ Clinical Medicine,
Journal Citation Reports/Science Edition, EMBase, Scopus and the Elsevier Bibliographic databases. The manuscript management system is completely online and includes a very quick and fair peer-review system, which is all easy to use. Visit http://www.dovepress.com/ testimonials.php to read real quotes from published authors.

Submit your manuscript here: http://www.dovepress.com/international-journal-of-nanomedicine-journal 